\title{
EFFECTS OF PARBOILING AND PHYSICO-CHEMICAL CHARACTERISTICS OF RICE ON THE GLYCEMIC AND INSULINEMIC INDICES IN TYPE 2 DIABETIC SUBJECTS
}

\author{
Shahana Parvin'1 , Qamrul Hasan², Knud Erik Bach Knudsen³, Liaquat Ali ${ }^{4}$ \\ ${ }^{1}$ Department of Biochemistry, Northern International Medical College, Dhaka \\ ${ }^{2}$ Department of Neurosurgery, Dhaka Medical College \& Hospital, Dhaka \\ ${ }^{3}$ Danish Institute of Agricultural, Sciences (DIAS), Denmark \\ ${ }^{4}$ Department of Biochemistry, Research Division, BIRDEM, Dhaka
}

\begin{abstract}
Background and Purposes: To observe the influence of parboiling, amylose content and gelatinization temperature of rice on plasma glucose and insulin responses in type 2 diabetic subjects because diabetic subjects are especially prescribed usage of starchy foods with low glycemic responses.

Methods: Seventeen type 2 diabetic subjects ingested five test meals of $50 \mathrm{~g}$ available carbohydrate as white bread, cooked rice with high (29\%) and low amylose content (13\%), undergoing different processing and gelatinization temperatures. The diets were taken in a random order after a $10 \mathrm{~h}$ overnight fast with approximately 7 days interval as wash out period.

Results: The glycemic index (GI) of all rice varieties were lower than that of white bread $(\mathrm{p}<0.001)$. Furthermore, GI of parboiled rice with a high amylose content was lower than that of parboiled low amylose rice $(50 \pm 7$ vs $71 \pm 5, p<0.01)$. No differences were observed between parboiled rice with high and low gelatinization temperature ( $50 \pm 7$ vs $47 \pm 4$ ), nor between non-parboiled and parboiled rice ( $52 \pm 7$ vs $50 \pm 7$ ). Insulin responses to the five test foods did not differ significantly in the study subjects.

Conclusions: In type 2 diabetic subjects the investigated rices were all low glycemic as compared to white bread, independent of parboiling and physico-chemical characteristics. The study showed that the amylose content, but not the gelatinization temperature, may be an useful criteria in selection of low GI rices irrespective of parboiling status.
\end{abstract}

Ibrahim Med. Coll. J. 2008; 2(1): 12-16

Key words: Amylose, blood glucose, insulin, type 2 diabetes, parboiled rice.

\section{Introduction}

Rice, the staple food, constituting up to $80 \%$ of the daily energy intake, is the main carbohydrate source of Bangladeshi population and is predominant in the daily diet for these people ${ }^{1}$. Diabetic subjects are especially prescribed starchy foods with low glycemic responses ${ }^{2}$. Wide variations have been observed in the glycemic responses to rice. Some studies have found a low glycemic response for rice compared to white bread $^{3}$. In contrast, Miller found higher glycemic responses to rice than to white bread ${ }^{4}$.
To prepare a diet plan for diabetic and cardiovascular patients in the local perspective, foods need to be graded according to their metabolic (glycemic and insulinemic) responses and physico-chemical properties. No such attempt has so far been made in this direction for Bangladeshi foods. The present work was performed to investigate three varieties of commonly consumed rice (BR16, BR25 and BR32) with varying processing (parboiled and non-parboiled), amylose content and gelatinization temperature in type 2 diabetic subjects.

Address for Correspondence:

Dr. Shahana Parvin, Associate Professor, Department of Biochemistry; Northern International Medical College; Plot \# 8A, Road \# 7, Dhanmondi; Dhaka - 1205, Bangladesh, Phone: 880-2-9668018, 8621479 - 83; Ext - 228 
The aim of the study was to examine the relationship between parboiling and physico-chemical characteristics of different rice varieties and the impact on blood glucose and insulin responses in type 2 diabetic subjects.

\section{Materials and Methods}

\section{Subjects}

Seventeen type 2 diabetic subjects ( 8 male, 9 female) were included in this study. The subjects were selected from the out-patients department of BIRDEM. Diabetes was diagnosed and classified by the WHO criteria ${ }^{5}$. Patients with acute or chronic complications of diabetes mellitus and those using insulin, oral contraceptives or steroids were excluded from the study. Pregnancy was also an exclusion criterion. All participants gave their written consent after being fully informed about the nature of the study.

\section{Methods}

Five test meals were served in a random order after an 8-10h overnight fast. An intravenous cannula was inserted into an antecubital vein in the forearm through which fasting blood samples were drawn and meals were served. The meals were consumed within 15 mins, along with $200 \mathrm{ml}$ of drinking water. Blood samples were then collected at 15, 30, 45, 60, 90, 120, 150 and 180 mins. Separated plasma was preserved at $-70^{\circ} \mathrm{c}$ prior to biochemical analysis.

Four varieties of rice and white bread as reference food, having $50 \mathrm{~g}$ available carbohydrate were given to the subjects. The rice varieties (BR16, BR25 and BR32) were obtained from Bangladesh Rice Research Institute (BRRI), Gazipur, where they were grown, harvested, parboiled, husked and milled. White bread was baked in one batch, sliced and portioned. Each bread portion was kept frozen and removed from the freeze $45 \mathrm{mins}$ before serving. The rice was boiled in excess water and cooked to its minimum cooking time to ensure the same degree of gelatinization of the starch.

\section{Physico-chemical characteristics of test food}

All physico-chemical properties were determined at BRRI. Grain shape was differentiated by length/breath ratio. Apparent amylose content was obtained by the iodine-blue colorimetric method ${ }^{6}$. Alkali spreading value was measured according to Little et al., which is an indicator of the gelatinization temperature (GT) and is inversely correlated to $\mathrm{GT}^{7}$. Equilibrium water content (EWC) was used as an indicator of the severity of parboiling. EWC was determined by weighing the water absorption in milled rice during a period of $23 \mathrm{~h}$ at $28-30^{\circ} \mathrm{C}$. Minimum cooking time was estimated as the time when $\geq 90 \%$ of the cooked rice grains had fully cooked centers.

\section{Chemical composition of test meals}

All chemical analysis was determined in duplicates at the National Institute of Animal Science, Foulum, Denmark. Dry matter (DM) was determined by oven drying at $105^{\circ} \mathrm{C}$ for $20 \mathrm{~h}$. Available carbohydrate was determined as total starch by an enzymatic colorimetric method ${ }^{8}$. Protein $\left(N^{*} 6.25\right)$ was analyzed using a Kjell-Foss 16200 Autoanalyser and fat was determined after hydrolysis and diethyl ether extraction according to Stoldt ${ }^{9}$.

\section{Biochemical analysis}

Glucose was measured by glucose-oxidase method (SERA PAK, USA); $\mathrm{HbA}_{1} \mathrm{c}$ by HPLC method (BIO RAD, USA) and plasma insulin was measured by a micro particle enhanced fluorescent immunoassay (EIMA) technique (Abbott Laboratories, USA).

\section{Statistical Analysis}

The incremental plasma glucose and insulin response areas were calculated geometrically according to Wolever and Jenkins ${ }^{10}$. Results were expressed as means \pm SD. Data were analyzed by a two way analysis of variance (ANOVA) followed by paired t-tests of means if the ANOVA indicated significance. The limit of significance was set at $\mathrm{p}<0.05$.

\section{Results}

The study was conducted on subjects (BMI $23 \pm 2 \mathrm{~kg}$ / $\mathrm{m}^{2}$, waist-hip ratio $1.03 \pm 0.02, \mathrm{M} \pm \mathrm{SD}$ ) with mild to moderate hyperglycemia $\left(\mathrm{M} \pm \mathrm{SD}, \mathrm{HbA}_{1} \mathrm{c} 7.07\right.$ $\pm 0.7 \%$, fasting glucose value $7.0 \pm 2.5 \mathrm{mmol} / \mathrm{L})$. The age of the subjects was $(42 \pm 5 \mathrm{y}, \mathrm{M} \pm \mathrm{SD}$ ) (Table 1$)$.

The glucose incremental response areas to all rice varieties were lower compared to white bread $(p<0.001)$. The same was reflected in their GI values. 
Table-1: Clinical and sociodemographic characteristics of the study subjects

\begin{tabular}{|c|c|}
\hline Variables & Values \\
\hline Age (years, $M \pm S D$ ) & $42 \pm 5$ \\
\hline $\mathrm{BMI}(\mathrm{M} \pm \mathrm{SD})$ & $23 \pm 2$ \\
\hline Waist-hip ratio (M $\pm \mathrm{SD})$ & $1.03 \pm 0.02$ \\
\hline Female : Male & $52: 48$ \\
\hline Rural : Urban & $3: 14$ \\
\hline Duration of diabetes (months- M $\pm S D$ ) & $6.2 \pm 3$ \\
\hline Fasting plasma glucose (mmol/L, M $\pm S D$ ) & $7.0 \pm 2.5$ \\
\hline Annual income (median-range) in US Dollars & $2068(1034-6206)$ \\
\hline $\mathrm{HbA}_{1 \mathrm{C}}(\%, \mathrm{M} \pm \mathrm{SD})$ & $7.7 \pm 0.7$ \\
\hline
\end{tabular}

Data expressed in $M \pm S D$, except for annual income, which is expressed in median (range).

The low amylose rice BR25pb had a very high glycemic index $(71 \pm 5)$ compared to other rice varieties (GI 50). The incremental insulin response areas to the rice meals also tended to be lower than to white bread. However, the difference was not significant statistically $(\mathrm{p}=0.04)($ Table 3$)$.

\section{Parboiling of rice}

Non-parboiled and parboiled rice of the same variety did not show statistical difference in incremental glucose and insulin response areas (Table 3). This was also reflected in their GI values $(50 \pm 7$ for BR $16 \mathrm{pb}$ and $52 \pm 7$ for BR16np) (Table 3).

Table-2: Physico-chemical characteristics of rice varieties

\begin{tabular}{lcccc}
\hline Rice variety & BR16np & BR16pb & BR25pb & BR32pb \\
\hline Length/breath ratio & 3.4 & 3.3 & 3.1 & 2.9 \\
Apparent Amylose content (\%) & 29 & 29 & 13 & 27 \\
GT / Alkali spreading value & 4 & 4 & 3 & 7 \\
Gel consistency? (mm) & 70 & 70 & 88 & 27 \\
Equilibrium water content (\%) & 11 & 26 & 28 & 28 \\
Minimum cooking time (min) & 17 & 22 & 20 & 21 \\
\hline
\end{tabular}

${ }^{*}$ Percentage of raw sample; 'Determined on non-parboiled samples; np: non-parboiled; pb: parboiled; Apparent amylose content: high ( $>25 \%)$, intermediate (20-25\%), low ( $<20 \%)$; Alkali spreading value (gelatinization temperature): high (1-3), intermediate (4-5), low (6-7)

Gel consistency: hard $(<40 \mathrm{~mm})$, medium $(41-60 \mathrm{~mm})$, soft $(>61 \mathrm{~mm})$; Length/breath ratio: long grain $(\geq 3.1)$, medium grain $(2.1-3.0)$, short grain $(\leq 2.0)$
Table-3: Metabolic responses to test meals $(n=17$ type 2 diabetic patients)

\begin{tabular}{lccccc}
\hline Test meal & WB & BR16np & BR16pb & BR25pb & BR32pb \\
\hline $\begin{array}{l}\text { P-glucose: iAUC } \\
\text { (mmol/L*180 min) }\end{array}$ & $756 \pm 65^{a}$ & $411 \pm 77^{\circ}$ & $391 \pm 69^{b}$ & $566 \pm 81^{c}$ & $361 \pm 48^{b}$ \\
Gl & $100^{\mathrm{a}}$ & $52 \pm 7^{\mathrm{b}}$ & $50 \pm 7^{\mathrm{b}}$ & $71 \pm 5^{\mathrm{c}}$ & $47 \pm 4^{\mathrm{b}}$ \\
Insulin: iAUC & $20184 \pm 1643$ & $1078 \pm 1268$ & $12811 \pm 1505$ & $16125 \pm 2983$ & $13011 \pm 1949$ \\
(?u/ml *180 min) & & & & & \\
\hline
\end{tabular}

Results are expressed as mean $\pm \mathrm{SD}$. $\mathrm{P}<0.05$ was taken as the level of significance. iAUC: Incremental area under cure; GI: glycemic index; WB: white bread. Means in the same row followed by different superscript letters are significantly different.

\section{Amylose content}

High amylose rice (BR16pb) showed a markedly lower

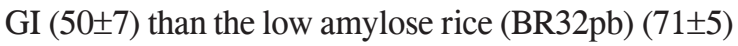
$(\mathrm{p}<0.01)$, while the incremental insulin response areas did not differ significantly (Table 2, 3).

\section{Gelatinization Temperature (GT)}

Rice with high GT (BR16pb) and low GT (BR25pb) showed no significant differences in mean incremental glycemic and insulinemic response areas (data not shown).

\section{Discussion}

Rice is suitable for use as low glycemic diets in the dietary management of type 2 diabetic subjects. Interestingly it is widely believed particularly in Asia like Bangladesh that diabetic subjects should limit their rice intake due to a positive association between a high intake of rice and the risk of developing diabetes. As starch is the principal component of rice, the physicians and dieticians advise diabetics as well as cardiovascular patients with substantial restriction of this major carbohydrate source. To rationalize the advice, it is important to know their physicochemical properties and their biological responses. The substitution of calories and other nutrients may then be done on the basis of patient's choice, socioeconomic capability and availability in the market.

Glycemic Index (GI) is a useful tool in measuring the biological response of a food in relation to glycemic status. The present study evaluates the GI of rice of varying physicochemical properties in type 2 diabetic subjects. In contrast to the popular impression, the results suggest that, rice varieties produces lower 
glycemic responses compared to white bread and this needs to be kept in mind when providing dietary advice to diabetic patients and calculating their calorie requirements. The rice varieties (BR16, BR25 and BR32) cooked for the same length of time, BR25 produce higher GI than other rice varieties. The results clearly demonstrated that the rice varieties investigated in this study, independent of processing and physico-chemical properties were low glycemic as compared to white bread. Thus, the rice with high or low amylose content, independent of processing is better recommendable than refined western bread as carbohydrate source in the diabetic diet.

The low GI of rice may be due to a delayed enzymatic hydrolysis of the whole grains, a process that can be accelerated by grinding ${ }^{11}$. In contrast Miller et al. found high GI to a number of Australian rice varieties ${ }^{4}$. These discrepancies may be due to differences in the physico-chemical characteristics, processing and/or cooking time of the rice varieties. Differences in the cooking time may influence the degree of gelatinization of the rice starch and the glycemic responses ${ }^{12}$. In the present study, the minimum cooking time for the rice was estimated and applied, ensuring that $\geq 90 \%$ of the rice kernels have full cooked centers. Thus, the low GI of rice in type 2 diabetic subjects found in the present study cannot be explained by the cooking time.

Insulin is the central hormone in maintaining blood glucose homeostasis. In type 2 diabetes mellitus the absolute level of insulin may be low, normal or higher in the blood although there is always a relative insulin deficiency. In spite of life saving role of the hormone, the higher level of insulin in blood (hyperinsulinemia) has been shown to be associated with increased atherosclerosis leading to cardiovascular disorders ${ }^{13}$. Due to this atherogenic role of insulin it is desirable to control the blood glucose of patients keeping the insulin levels as normal as possible. In this context, plasma insulin measurement in the present study has an important implication. The results showed that the insulin responses also did not differ significantly among the test foods. So, we can state that the insulin secretory capacity was not influenced by the test foods.

The study found no effect of parboiling on plasma glucose and insulin responses as well as in the GI values. This is in accordance with the results of Miller, but in contrast to Casiragi ${ }^{4}, 14$. One explanation for the varying effects may be ascribed to the parboiling process used. In our study, a traditional parboiling process, adapted from BRRI, was applied. This method may be regarded as a relatively mild procedure compared to the parboiling process used in the industrial trade, e.g. pressure parboiling. The severity of parboiling has been shown to affect some of the physico-chemical properties of rice starch ${ }^{15}$.

We found that high amylose (29\%) rice lower plasma glucose response and GI values compared with low amylose (13\%) rice in type 2 diabetic patients. This finding is in agreement with the results of previous studies ${ }^{16,4}$. The effect of amylose on the glycemic and insulin responses may partly be explained by the structure of the starch. Starch granules rich in amylose have extensive hydrogen bonding due to the linear structure of amylose which restricts the rate and degree of swelling and gelatinization of the starch during thermal processing, thereby slowing down the starch digestion and absorption rate ${ }^{17}$. Another explanation for the lower GI measured in high amylose rice could be the increased number of starch-lipid complexes in the cooked rice ${ }^{18}$.

Panlasigui et al. suggested that GT might be a useful parameter to predict the variation in the metabolic responses observed for rices with similar amylose content ${ }^{12}$. From the study we found no differences in the plasma glucose and insulin responses in the study subjects after ingestion of parboiled rice with low and high GT. A number of reasons may explain this result. We cooked the rice samples to the estimated minimum cooking time. It is also possible that the parboiling process reduced a possible effect of GT on the glycemic and insulinemic responses. Finally, the two rice varieties varied in gel consistency, which may have acted as a confounding factor.

\section{Conclusions}

The rice analyzed are low glycemic in type 2 diabetic subjects, independent of parboiling and physicochemical properties. Thus, the rices are suitable for use as low glycemic diets having beneficial properties in the dietary management of type 2 diabetic subjects. The study showed that the amylose content, but not the GT, may influence GI of parboiled rice, and therefore be a useful criterion for selection of low GI rices also after parboiling. The mild parboiling method used in this study did not affect the GI, but indicated the need for further investigations on the influence of the severity of parboiling on GI. 


\section{References}

1. Choudhury OH. A review of literature on nutrition studies in Bangladesh. Dhaka: Bangladesh Institute of Development Studies, 1992.

2. Pedersen O, Hermansen K, Palmvig B, Pedersen SE and Sondergaard K. Danish Diabetic Association: Rationale for diet recommendations in the 1990's. Scand J Nutr 1994; 38: 129-133.

3. Jenkins DJA, Wolever TMS, Tailor RH, Barker H, Fielden H, Baldwin JM et al: Glycemic Index of foods: a physiological basis for carbohydrate exchange. Am J Clin Nutri 1981; 34: 362-366.

4. Miller JB, Pang E and Barmall L. Rice: a high or a low glycemic index food. Am J Clin Nutr 1992; 56: 1034-1036.

5. WHO Study Group. Prevention of diabetes mellitus. WHO Technical Report Series no 844. World Health Organization, Geneva, 1994.

6. Juliano BO, Perez CM, Blakeney AB, Castillo DT, Kongseree N, Laignelet B et al. International cooperative testing on the amylose content of milled rice. Starch 1991; 33: 157-162.

7. Little RR, Hilder GB and Dawson EH. Differential effect of dilute alkali on 25 varieties of milled white rice. Cereal Chemists 1985; 35: 111-126.

8. Bach Knudsen KE, Aaman P and Eggum BO. Nutritive values of Danish-grown barley varieties. Carbohydrates and other major constituents. J Cereal Science 1987; 6: $173-186$.

9. Stoldt W. Suggestions to standardize the determination of fat in foodstuffs. Fette, seifen, anstrichtsmitted 1952; 54: 206-207.
10. Wolever TMS and Jenkins DJA. The use of glycemic index in predicting the blood glucose response to mixed meals. Am J Clin Nutr 1986; 43: 167-172.

11. O'Dea K, Nestal PJ and Antonoff L. Physical factors influencing postprandial glucose and insulin responses to starch. Am J Clin Nutr 1980; 33: 760-765.

12. Palasigui LN, Thompson LU, Juliano BO, Perej CM, Yiu SH and Greenberg GR. Rice varieties with similar amylose content differ in starch digestibility and glycemic response in humans. Am J Clin Nutr 1991; 54: 871-877.

13. Kaplan NM. Hypertension and diabetes. In: Porte D Jr, Sherwin RS, editors. Ellenberg and Rifkin's Diabetes Mellitus. $5^{\text {th }}$ ed. Stamford Connecticut: Appleton and Lange 1996; 1097-1104.

14. Casiraghi MG, Brighenti F, Pellegrini P, Leopardi E and Testolin G. Effect of processing on rice starch digestibility evaluated by in vivo and in vitro methods. J Cereal Sci 1993; 17: 147-156.

15. Biswas SK and Juliano BO. Laboratory parboiling procedures and properties of parboiled rice from varieties differing in starch properties. Cereal Chemists 1988; 65: $417-423$.

16. Goddard MS, Young G and Marcus R. The effect of amylose content on insulin and glucose responses to ingested rice. Am J Clin Nutr 1984; 39: 388-392.

17. Sowbhagya CV, Ramesh BS and Ali SZ. Hydration, Swelling and solubility behavior of rice in relation to other physico-chemical properties. J Sci Food Agric 1994; 64: 1-7.

18. Tester RF and Morrison WR. Swelling and gelatinization of cereal starches. Effects of amylopectin, amylose and lipids. Cereal Chem 1990; 67: 551-557. 